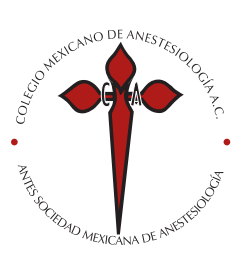

\title{
Impacto de la anestesia intravenosa en cirugía robótica para paciente de alto riesgo sometido a cirugía oncológica de larga duración en el Centro Médico Naval
}

\author{
Impact of intravenous anesthesia in robotic surgery for high-risk patient \\ undergoing long-term oncologic surgery at the Naval Medical Center
}

\section{Dra. Verónica Guadarrama-Bustamante,* Dr. Juan Alberto Díaz-Ponce-Medrano, ${ }^{\ddagger}$ Dr. José Carlos Buenfil-Medina, ${ }^{\S}$ Dr. Juan Carlos Orozco-Lara, Dr. Víctor Manuel Antonio-Figueroa," Dr. Christian Isaac Villeda-Sandoval,** Dr. Víctor Hugo Villaseñor-Santiago, ${ }^{\#}$ Dr. Juan Heberto Muñoz-Cuevas ${ }^{\S \S}$}

Citar como: Guadarrama-Bustamante V, Díaz-Ponce-Medrano JA, Buenfil-Medina JC, Orozco-Lara JC, AntonioFigueroa VM, Villeda-Sandoval Cl, et al. Impacto de la anestesia intravenosa en cirugía robótica para paciente de alto riesgo sometido a cirugía oncológica de larga duración en el Centro Médico Naval. Rev Mex Anestesiol. 2021; 44 (3): 215-224. https://dx.doi.org/10.35366/99669

RESUMEN. La práctica de cirugía robótica es un proceso complejo que implica desarrollo y tecnología no sólo en el campo de la cirugía, sino también en el campo de la anestesiología. Implica un proceso multifactorial, ya que ha generado un cambio drástico multidisciplinario basado en tecnología de punta que pretende ofrecer mejores condiciones durante el manejo perioperatorio en cirugía robótica. La anestesia total intravenosa cumple objetivos específicos en relación a la posición del paciente, relajación cerebral, neuroprotección, hemodinamia, pérdida y recuperación de la conciencia, parálisis neuromuscular, parámetros ventilatorios, etc. Ofrece seguridad y calidad al paciente durante el procedimiento con una mínima interferencia con el monitoreo electrofisiológico y permite modular la profundidad anestésica desde una neurosedación hasta una anestesia general, de acuerdo a las diferentes etapas de la cirugía. Un factor atribuible a la anestesia moderna para el éxito de la cirugía robótica es usar diferentes agentes anestésicos que promuevan inducción, mantenimiento y emersión anestésica más rápida y suave, a fin de reducir el tiempo de recuperación del estado de conciencia, funciones básicas y psicomotoras como la anestesia general multimodal.

ABSTRACT. The practice of robotic surgery is a complex process, involving development and technology; not only in the surgery field but also in the anesthesiology field. It implies a multifactorial process since it has generated a drastic multidisciplinary change based on state-of-the-art technology; which aims to offer better conditions during perioperative management in robotic surgery. Intravenous Total Anesthesia accomplishes specific objectives in relation to patient position, brain relaxation, neuroprotection, hemodynamics, loss and recovery of consciousness, neuromuscular paralysis, ventilatory parameters, providing safety and quality during the procedure; with minimal intervention during electrophysiological monitoring and enabling anesthetic depth to be modulated from neurosedation to general anesthesia, according to the different stages of the surgery. A factor attributable to modern anesthesia for robotic surgery success is to employ different anesthetic agents promoting induction, maintenance of general anesthesia, smother and faster anesthetic emersion, for the purpose of reducing recovery time of the state of consciousness), basic and psychomotor functions; as is the general multimodal anesthesia.

SIGLAS:

ECG $=$ Electrocardiograma.

PANI = Presión arterial no invasiva.

BIS = Índice biespectral.

EEG = Electroencefalograma

NIRS = Noxius impulse response index .

TNM o TOF $=$ Tren de cuatro o train of four:

MNM = Monitoreo neuromuscular.

BNM = Bloqueadores neuromusculares.

ATIV = Anestesia total intravenosa.

TCI = Target controlled infusion .
$\mathrm{EtCO}_{2}=$ Dióxido de carbono teleespirado.

$\mathrm{Ce}=$ Concentración en sitio efecto.

$\mathrm{DC}=$ Dosis de carga o bolo inicial.

MIR = Velocidad de perfusión manual

para mantenimiento anestésico.

IV = Intravenoso.

Cp = Concentración plasmática.

$\mathrm{Cl}=$ Clearance.

$\mathrm{Vd}=$ Volumen de distribución central.

$\mathrm{T} 3 \mathrm{~b}=$ Tumor que invade los tejidos que rodean la vejiga con invasión macroscópica. E-mail: veronicaguadarrama750@yahoo.com
N1 = Metástasis en un solo ganglio linfático de $2 \mathrm{~cm}$ o menor. $\mathrm{DO}_{2}=$ Aporte de Oxígeno Correspondencia: Dra. Verónica Guadarrama Bustamante Departamento de Anestesiología. Centro Médico Naval. Secretaría de Marina Armada de México. Eje 2 Oriente. Tramo Heroica Escuela Naval Núm. 701, Col. Presidentes Ejidales, 04470, Alcaldía Coyoacán, Ciudad de México.
Palabras clave: Robot Da Vinci Xi, cirugía robótica, cáncer de vejiga, BIS, NIRS, TNM, anestesia total intravenosa anestesia general multimodal.

Keywords: Da Vinci Surgical robot Xi, robotic surgery, bladder cancer, BIS, NIRS, TOF, total intravenous anesthesia, multimodal general anesthesia.

*Anestesióloga Oncóloga Naval. Profesor titular de la Especialidad de Anestesiología. Médico Adscrito. Centro Médico Naval. Secretaría de Marina-Armada de México. Miembro de Tiva México. Miembro de Tivamérica.

${ }^{\ddagger}$ Médico Cirujano Naval. Anestesiólogo Pediatra. Director del Centro Médico Naval. Secretaría de Marina-Armada de México.

$\S$ Jefe de Enseñanza, Investigación y Calidad. Centro Médico Naval. Secretaría de MarinaArmada de México.

` Médico Cirujano Naval Urólogo. Jefe de Área Médica. Centro Médico Naval. Secretaría de Marina-Armada de México. " Médico Cirujano Naval. Anestesiólogo en Trasplantes. Jefe del Apoyo Médico. Centro Médico Naval. Secretaría de Marina-Armada de México. ** Médico Cirujano Urólogo Oncólogo. Coordinador de tumores genitourinarios y del Comité de Cirugía Robótica.

‡‡ Médico Cirujano Naval. R1 de Anestesiología. Centro Médico Naval. Secretaría de Marina-Armada de México.

$\S \S$ Médico Anestesiólogo.

Coordinador Tiva México.

Miembro de Tivamérica.

Recibido: 22-04-2019

Aceptado: 17-07-2020 


\section{INTRODUCCIÓN}

$\mathbf{R}$ eporte de un caso de cistectomía radical y linfadenectomía pélvica bilateral superextendida con neovejiga intracorpórea laparoscópica asistida con robot Da Vinci Xi, con duración de más de 15 horas, bajo anestesia total intravenosa multimodal con titulación de los agentes anestésicos en modo TCI Schnider para propofol y modo manual para opioide y coadyuvantes, realizada en el Centro Médico Naval de la Secretaría de Marina Armada de México (Figura 1).

El cáncer de vejiga se ha convertido en el cuarto cáncer más común entre la población mexicana. Aún poco estudiado es cuatro veces más frecuente entre hombres que entre mujeres y $90 \%$ de los casos se presenta en adultos mayores de 55 años. El tipo más frecuente de cáncer de vejiga (90\%) es el carcinoma de células transicionales, este tipo de cáncer comienza en las células que normalmente forman el revestimiento interior de la vejiga, también llamado epitelio ${ }^{(1)}$. El tratamiento estándar para el cáncer de vejiga con invasión muscular incluye la cistectomía radical. Para los pacientes varones, además de la extirpación completa de la vejiga y todo tejido tumoral posible, también implica extirpación de la uretra, próstata, vesículas seminales, partes inferiores de los uréteres y los ganglios linfáticos en la pelvis. La cistectomía radical lleva a la pérdida de la función vesical, por ello el cirujano realiza una derivación urinaria ${ }^{(2)}$.

El robot Da Vinci Xi consiste en una plataforma robótica sofisticada y única en el mundo. Está diseñado y fabricado por la empresa norteamericana Intuitive Surgical y tiene el objetivo de potenciar las capacidades y habilidades del cirujano así como de ofrecer una opción mínimamente invasiva en procedimientos de mayor complejidad quirúrgica. El robot Da Vinci Xi es actualmente la plataforma más evolucionada destinada a la cirugía mínimamente invasiva. Su tecnología innovadora y precisa dota a este sistema robótico de adaptabilidad y versatilidad. El robot Da Vinci Xi (IS4000) permite entre otras cosas: realizar un aumento de hasta 10 veces del campo operatorio con una resolución detallada indudablemente superior a la técnica laparoscópica, anular el temblor de las manos del cirujano e impedir movimientos involuntarios, intercambiar los cuatro brazos robóticos de los que está provisto, interactuar con otras tecnologías normalmente presentes en los quirófanos de todo el mundo, optimizar mediante un sistema láser la posición de los brazos robóticos según la intervención establecida y la posición de los trócares, usar instrumentos con un diámetro de 8-12 mm llamados Endowrist capaces de realizar un movimiento de casi $360^{\circ}$ sobre siete ejes, practicar cirugía multicuadrante e incluso operar sobre varios órganos situados en diferentes cuadrantes anatómicos sin mover al paciente o al robot, tener una segunda consola útil para la colaboración de otros cirujanos durante la intervención con óptimos resultados en términos de formación, desarrollado con base en el concepto de la Immersive Intuitive Interface. Es el único sistema robótico que traduce los movimientos del cirujano de manera intuitiva, permitiendo un control total de la fibra óptica y de los instrumentos evitando complejos movimientos laparoscópicos, está dotado de un sistema de posicionamiento por láser que permite optimizar el posicionamiento de los brazos en función del tipo de intervención seleccionado y de la posición de los trócares para reducir el tiempo de preparación y de operación.

La cirugía robótica proporciona múltiples beneficios, entre los cuales cabe destacar menor morbilidad, control oncológico radical, facilidad de acceso a anatomías complicadas, visualización más detallada de los planos de hendidura y la posibilidad de configurar la precisión del movimiento quirúrgico.

La cirugía robótica, también conocida como cirugía laparoscópica asistida por robot, es una técnica mediante la cual el cirujano certificado lleva a cabo la cirugía usando una consola que controla de manera remota instrumentos muy pequeños fijados a un robot. Su aplicabilidad ha quedado demostrada en procedimientos complejos como esofagectomías, neumonectomías, pancreatectomías, cirugía cardiovascular y cardiotorácica, cirugía de la obesidad, cirugía urooncológica, cirugía ginecooncológica, cirugía oncológica, cirugía general y cirugía pediátrica.

Dentro de las ventajas para el paciente: las incisiones son pequeñas con mejores resultados estéticos, hay menor necesidad de transfusiones, menos dolor postoperatorio, reducción del tiempo de hospitalización, incorporación rápida a las actividades del paciente, alteración menos expresiva en los valores del hematocrito. El grado de dificultad para el manejo anestésico se incrementa, ya que es imperativo invadir al paciente con el monitoreo necesario previo al evento quirúrgico, dependiendo de las condiciones clínicas en las que

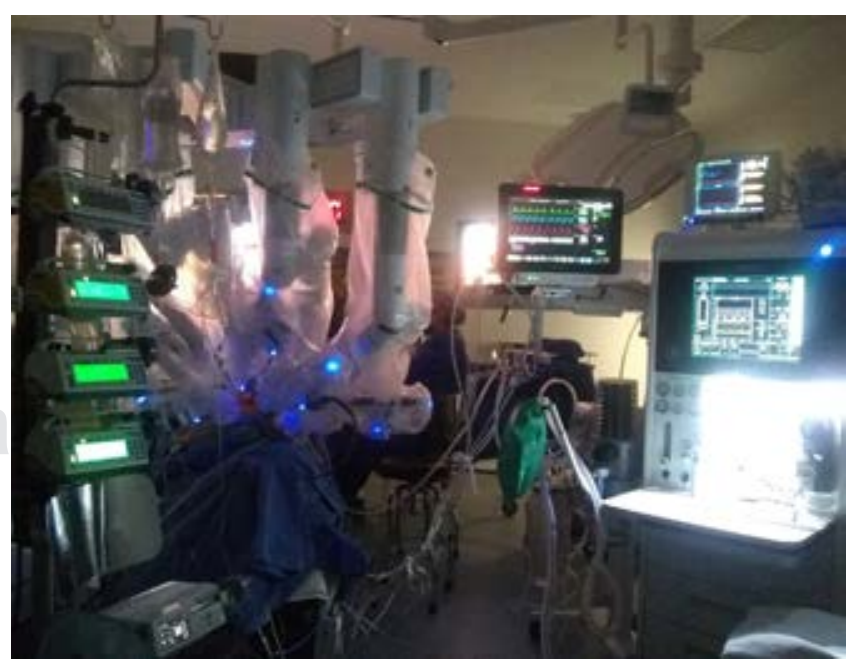

Figura 1: Anestesia total intravenosa multimodal con neuromonitoreo. 
se encuentre el paciente (monitoreo tipo I y monitoreo tipo II). La posición que adopta el paciente durante la cirugía es con los brazos a los costados del cuerpo para que los brazos robóticos puedan abordar al paciente durante la cirugía. La posición de Trendelenburg es muy forzada, aproximadamente de $60^{\circ}$ a $84^{\circ}$ de inclinación, la posición del paciente debe ser estrechamente vigilada para evitar cualquier daño neurológico, artralgia o lesión digital, los hombros y los pies deben quedar apoyados adecuadamente y el tórax debe quedar fijo sin que su expansión quede comprometida durante la ventilación.

Es absolutamente necesario como anestesiólogos conocer las alteraciones fisiológicas asociadas a procedimientos quirúrgicos robóticos, considerar los cambios a nivel cardiopulmonar cuando los pacientes se colocan en la posición de Trendelenburg forzada y se genera el neumoperitoneo, considerar las presiones intraabdominales elevadas y el tiempo quirúrgico ${ }^{(3,4)}$.

En el contexto actual de la anestesiología hacia la medicina perioperatoria en cuanto a la optimización de los pacientes durante todo el proceso quirúrgico es esencial incluir el control de la respuesta al estrés quirúrgico relacionada con la morbimortalidad perioperatoria y con el dolor crónico postoperatorio, independientemente del tiempo quirúrgico que se requiera. Esta respuesta va a implicar la liberación hormonal de mediadores inmunológicos, y la activación del sistema nervioso simpático. Conocer estos tres componentes: endocrino, inmunológico y del sistema nervioso autónomo será imprescindible para monitorear y tratar de bloquear dicha respuesta. Durante la cirugía se requieren condiciones hemodinámicas estables para mantener el balance de la oxigenación miocárdica y cerebral, toda vez que la taquicardia y la hipertensión arterial son factores de riesgo de isquemia miocárdica.

El monitoreo de la pérdida y recuperación de la conciencia permite evaluar de manera muy objetiva el estado de alerta de un paciente y los efectos cerebrales de los anestésicos. El índice biespectral BIS es un parámetro de monitoreo de profundidad anestésica de los más utilizados y validados desde los años 90 hasta la actualidad. Es una interpretación estadística basada en un algoritmo matemático complejo sobre datos extraídos del EEG del paciente sometido a anestesia general inhalada, balanceada y ATIV, que calcula un valor resultante de procesar una señal de electroencefalografía frontal. Dicho valor adimensional proporciona un algoritmo numérico que se correlaciona para evaluar la actividad cerebral del nivel del paciente. Valora los cambios que se producen en el trazo electroencefalográfico de forma cronológica. Pertenece a este análisis la tasa de supresión. La TS calcula la relación entre los períodos con presencia de señal en el EEG y los períodos en que aparece el trazado isoeléctrico en el último minuto (potencia no supera $\pm 5 \mu \mathrm{v}$ ). En casos de anestesia muy profunda se correlaciona con el BIS (BIS 0-40). El valor de la
TS es 0 en individuos despiertos, sedados o con una técnica anestésica transoperatoria adecuada. La aparición de la tasa de supresión en estos casos puede constituir una señal de isquemia cortical. En los casos de muerte encefálica el BIS es de 0 y la TS es de $100^{(5)}$.

El NIRS nos va permitir reflejar el comportamiento eléctrico cerebral en respuesta a los hipnóticos y al efecto de los estímulos nociceptivos de diferente intensidad que tiene lugar durante la intervención quirúrgica. La oximetría cerebral es una técnica no invasiva para monitorear cambios en el metabolismo cerebral de oxígeno. El método se basa en la tecnología NIRS near infrared spectroscopy. Se emiten fotones de luz cercanos al infrarrojo «NIR» en la piel de la frente del paciente. Después de dispersarse por el interior del cuero cabelludo, cráneo y cerebro, parte de estos fotones vuelven a la piel por reflectancia. Al medir la cantidad de fotones que regresan se puede inferir la absorción espectral del tejido subyacente y sacar conclusiones sobre su oxigenación media. $\mathrm{Al}$ dispersarse por los tejidos estos fotones son absorbidos en parte, y dependiendo de su longitud de onda, por los materiales coloreados (cromóforos) allí presentes, produciendo unas señales características en el espectro de luz emergente. El cromóforo con mayor absorción de luz en el tejido corporal es la hemoglobina, cuyo espectro de absorción varía según su estado de oxigenación. Se utilizan dos sensores que se colocan a ambos lados de la línea media. En cada sensor hay un punto emisor de luz y dos puntos de detección de la señal, situados a 3 y $4 \mathrm{~cm}$ del punto emisor. La fuente de luz emite dos haces de luz en el rango próximo al infrarrojo (730 y 810 $\mathrm{nm}$ ) e ilumina el tejido. La intensidad de la luz que recibe cada detector se convierte en una señal eléctrica que se procesa y digitaliza, y en pantalla aparecen los valores de $\mathrm{rSO}_{2}$ de los dos hemisferios cerebrales actualizados cada cuatro segundos. En la parte izquierda de la pantalla aparecen las tendencias. El lecho vascular cerebral está formado principalmente por vasos para el intercambio de gases, sobre todo vénulas. A pesar de las variaciones interindividuales se asume que $25 \%$ son vasos arteriales y $75 \%$ venosos, aunque los cambios en esta relación 25:75 influyen poco en la interpretación clínica de la $\mathrm{rSO}_{2}$. Por tanto, los valores obtenidos de $\mathrm{rSO}_{2}$ representan fundamentalmente el estado de oxigenación de los cromóforos del lecho vascular cerebral del compartimento venoso. Los cambios en la oximetría cerebral dependen del balance entre aporte y consumo de oxígeno (Figura 2). Los sensores se colocan en la frente del paciente, a ambos lados de la línea media, intentando evitar el seno sagital superior. La zona que se monitorea es la región perfundida por las arterias cerebrales media y anterior. La anatomía vascular cerebral en estas regiones las hace particularmente vulnerables a las deficiencias de aporte de oxígeno. La variación interindividual de los valores normales de la $\mathrm{rSO}_{2}$ es muy amplia, entre 20 y 36 años los valores obtenidos fueron $71 \pm 6 \%$ (rango 58-82). 


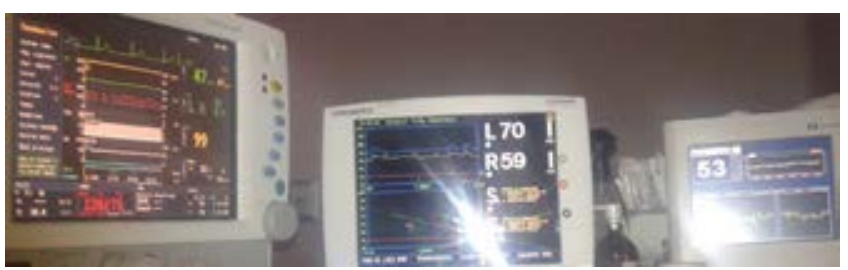

Figura 2: Monitoreo hemodinámico y oxigenación cerebral.

En pacientes que van a ser intervenidos en edad de 21-91 años los valores obtenidos fueron $67 \pm 10 \%$ (rango 47-83). Debido a estas variaciones se recomienda utilizarlo como un monitoreo de tendencias. La saturación basal es el valor inicial de $\mathrm{rSO}_{2}$ y sobre él se compararán los siguientes valores. El valor basal se obtiene con el paciente acostado y en reposo antes de ser anestesiado ${ }^{(6,7)}$.

Los objetivos del monitoreo del uso de bloqueadores neuromusculares (BNM) deben estar basados en criterios de efectividad, es decir, administrar la mínima dosis efectiva y en criterios de seguridad, evitando la sobredosificación y detectando el posible bloqueo residual antes de la extubación de los pacientes. El monitoreo perioperatorio de la función neuromuscular, junto con la detección de movimientos musculares, permite ajustar la dosis a la mínima efectiva para alcanzar el efecto clínico deseado, laringoscopía, intubación orotraqueal, posición en la mesa quirúrgica, ventilación controlada, insuflación del neumoperitoneo, colocación y movimiento del robot hasta el final de la cirugía permitiendo una recuperación del efecto del BNM minimizando el riesgo de parálisis residual. La estimulación del nervio cubital con detección de las respuestas en el aductor del pulgar es el registro que más comúnmente se lleva a cabo en la práctica diaria.

Es importante considerar la farmacología del BNM a utilizar, ya que el perfil farmacocinético tanto de aminoesteroides y benzilisoquinolínicos tienen tasas de clearance plasmático menores de $5 \mathrm{~mL} / \mathrm{kg} / \mathrm{min}$ que aunado a factores como los cambios en el volumen de distribución, las alteraciones de la función renal y/o hepática, las alteraciones hidroelectrolíticas o metabólicas, la temperatura y la coadministración de otros fármacos modifican la respuesta a los BNM.

La administración de un BNM sin monitoreo adecuado puede predecir la intensidad del efecto, la duración del mismo y su eliminación para una recuperación adecuada de la función neuromuscular. Sin el uso racional de este monitoreo no es posible la detección de estos eventos en el período transanestésico, pudiendo asociarse a una acumulación del fármaco, retrasando la posibilidad de inicio de la ventilación espontánea, eficaz y eficiente en el postoperatorio inmediato. El monitoreo rutinario y el ajuste de la dosificación a un objetivo permiten identificar en qué momento se lleva a cabo la recuperación del boqueo neuromuscular una vez suspendido el $\mathrm{BNM}^{(8)}$.
Cada uno de los componentes del estado anestésico debe tener una prioridad dependiente de la situación clínica y controlada por el anestesiólogo, considerando como base que los efectos de los fármacos intravenosos dependen tanto de sus propiedades fármaco cinéticas como fármaco dinámicas.

La cirugía es muy importante dentro del tratamiento multimodal de los tumores sólidos. La propia manipulación quirúrgica facilita la diseminación y la aparición de metástasis clínicas. La función inmunológica, sobre todo la inmunidad celular a expensas de las células NK (Natural Killer), tiene un papel fundamental en la recidiva tumoral y en la supervivencia. Hay un interés creciente en los eventos que se producen durante el período perioperatorio que conducen a la liberación de las células cancerosas en la circulación y que propician el crecimiento de las células tumorales circulantes, dando lugar a recurrencias regionales y metástasis. De las muertes relacionadas con el cáncer, 90\% se deben a la progresión de la enfermedad, a las metástasis y no al tumor primario. Por ello, prevenir la inmunosupresión en el período perioperatorio toma particular importancia. Durante el período perioperatorio existen factores que activan o inhiben las respuestas inmunitarias. Los descubrimientos recientes han sugerido que la anestesia puede inducir cambios metabólicos, inflamatorios e inmunológicos en el período perioperatorio.

La técnica anestésica per se influye en los resultados oncológicos, los fármacos anestésicos son capaces de inducir cambios biomoleculares involucrados en las funciones fisiopatológicas celulares tales como la proliferación, la angiogénesis y la apoptosis celular, todos ellos decisivos en la progresión de la enfermedad. Los datos in vitro del posible papel prometastásico de los anestésicos inhalatorios se basan en sus efectos inhibitorios, tiempo y dosis dependiente de la función de los neutrófilos, la supresión de las citocinas liberadas por las células mononucleares, la disminución de la proliferación linfocitaria y el efecto favorecedor en la inducción de la apoptosis linfocitaria. Además de sus efectos en el sistema inmunológico, los anestésicos inhalatorios también promueven el crecimiento y proliferación de las células madre del endotelio (efecto proangiogénico) que propiciaría el crecimiento tumoral y/o la teragenicidad. Por ejemplo, el propofol con edetato favorece la citotoxicidad de las células NK, reduce la motilidad e invasividad de las células tumorales, inhibe la ciclooxigenasa y no promueve la síntesis, es decir, tiene un efecto antiangiogénico y de diferenciación de linfocitos T helper, tiene actividad inhibitoria de COX-2, liberación de interferón gamma y mayor atenuación de la respuesta al estrés. El uso de propofol con edetato revela un efecto favorable en el sistema inmunológico en términos de IL-2 /IL-4 y células T CD4+/CD8+ en el período perioperatorio. Con respecto a los leucocitos y células Killer (NK), el propofol con edetato tiene un efecto positivo en las células inmunes antitumorales y las propiedades frente 
a la proliferación y metástasis de las células cancerosas. El propofol tiene un papel inhibitorio en la glucolisis de células de cáncer colorrectal. Se sugiere además que inhibe la expresión de transportadores de glucosa y enzimas (GLUT1, HK2, LDHA, y PDK1) que son cruciales en el metabolismo de la glucosa. Más allá se mantuvo la hipótesis de que HIF1, un factor maestro de la transcripción que controla el metabolismo de glucosa, también se ve disminuido por el propofol en una manera dosis dependiente ${ }^{(9,10)}$.

El propofol puede tener efectos protectores mediados por inhibición de COX-2 y PGE-2 y favorece la actividad antitumoral, pero también mediante el incremento de la actividad antitumoral. El propofol es superior a los agentes volátiles en reducir el estrés quirúrgico que induce la supresión en la respuesta inmunitaria. Estos resultados dan pie a pensar que la TIVA es preferible a los agentes inhalados en pacientes intervenidos quirúrgicamente por cáncer. Además ejerce efectos antitumorales por la inhibición de la viabilidad de la célula, la dispersión y la capacidad de invasión de células cancerosas $^{(11,12)}$.

Los anestésicos inhalatorios generan un posible papel prometastásico que se basa en sus efectos inhibitorios tiempo y dosis-dependiente de la función de los neutrófilos, la supresión de citocinas liberadas por las células mononucleares, la disminución de la proliferación linfocitaria y el efecto favorecedor en la inducción de la apoptosis linfocitaria. Además de sus efectos en el sistema inmunológico, los anestésicos inhalatorios también promueven el crecimiento y proliferación de las células madre del endotelio (efecto proangiogénico) que propiciaría el crecimiento tumoral y/o la teratogenicidad ${ }^{(13)}$. Los anestésicos inhalatorios afectan la respuesta inmunitaria, disminuyen la actividad de las células NK e incrementan la expresión de la hipoxia inducible por el factor de hipoxia $1 \alpha$ (HIF-1 $\alpha$ ). El sevofluorano induce la apoptosis de linfocitos T y produce un incremento en los niveles de citoquinas protumorigénicas; el isoflurano y el desflurano atenúan la actividad de las células NK, inducen apoptosis de linfocitos T y B y disminuyen la respuesta Th1/Th2; el desflurano se diferencia porque no induce apoptosis de linfocitos $\mathrm{T}^{(14)}$.

Los opioides interfieren con la función inmunológica deprimiendo la actividad de las células NK; sin embargo, también los opioides pueden reducir la respuesta de estrés al dolor. Los opioides sintéticos (fentanilo) a concentraciones plasmáticas bajas no alteran la inmunidad celular y se minimiza el riesgo de complicaciones respiratorias postoperatorias correlacionadas al uso de dosis altas de estos fármacos ${ }^{(14)}$.

Los opioides estimulan la angiogénesis mediante la activación de ciclooxigenasa 2 (COX-2). Esto aumenta la producción de prostaglandina E2, que promueve la angiogénesis y afecta directamente el crecimiento del tumor, activación de vías de señalización periférica, siendo que la activación del receptor opioide desencadena múltiples eventos de seña- lización en sentido descendente que incluyen: disminución de monofosfato de adenosín cíclico (cAMP) aumentando el óxido nítrico y niveles de monofosfato de guanosina cíclico, estimula también la fosfolipasa $\mathrm{C}$ y proteína quinasa activada por mitógeno (MAPK) y proteína quinasa C. El fentanilo suprime la cantidad y función de las células NK, las cuales desempeñan un papel en la inmunidad innata y adaptativa, por lo que tras su empleo incrementa el riesgo de metástasis tumoral ${ }^{(15,16)}$.

Las citocinas inflamatorias regulan la expresión del gen del receptor $\mu$-opioide (MOR), destacando una interacción entre los sistemas opioide e inmunológico. Los opioides inhiben componentes de la inmunidad humoral mediada por células. Se demostró que el fentanilo $\left(40 \mu \mathrm{g} \mathrm{kg}^{-1}\right)$ una hora antes de una laparotomía deprime la función de las células NK en un modelo de rata. Se observó un rápido aumento en la actividad de las células NK durante las primeras 24 horas, pero esto fue seguido de una disminución significativa de la actividad, volviendo sólo a los niveles de actividad basales después de ocho días ${ }^{(17)}$.

La dexmedetomidina es un agonista del receptor adrenérgico alfa ${ }_{2}$ altamente selectivo, se usa cada vez más durante el período perioperatorio en pacientes oncológicos e induce sedación, analgesia, ansiolisis e inhibición del tono simpático. La dexmedetomidina puede suprimir el estrés y la inflamación y preservar la inmunidad de los pacientes quirúrgicos, protege la función inmunológica y exhibe efectos protectores multifacéticos cuando se administra como adyuvante de la anestesia. Todos estos datos indican que la dexmedetomidina puede brindar beneficios a los pacientes quirúrgicos durante el período perioperatorio y mejorar los resultados clínicos. La infusión de dexmedetomidina durante el período perioperatorio inhibe la liberación de epinefrina, norepinefrina y cortisol; la disminución de la glucosa en sangre, interleucina (IL)-6, factor de necrosis tumoral- $\alpha$ y proteína $\mathrm{C}$ reactiva; $\mathrm{y}$ el aumento de la interleucina-10 en pacientes quirúrgicos ${ }^{(18)}$.

El magnesio es el segundo catión intracelular más abundante en el organismo después del potasio. El magnesio tiene tres funciones básicas principales: 1 . En el metabolismo energético, 2. El paso de iones transmembrana, 3. La activación de numerosas enzimas, es antagonista del receptor NMDA (N-metil D-aspartato) del glutamato, principal neurotransmisor excitador, lo que explica los efectos sedantes y en la modulación algésica, en la médula bloquea las vías del dolor dependientes del glutamato, además es un antagonista del calcio, por lo que relaja la musculatura lisa. El antagonismo de los receptores de NMDA localizados en el asta posterior de la médula espinal por el sulfato de magnesio produce modulación de la percepción central del dolor, provenientes de los nervios periféricos, efecto que se traduce en mayor analgesia en 24 horas en pacientes postoperados de cirugía oncológica $^{(19)}$. 
La perfusión perioperatoria de la lidocaína intravenosa tiene propiedades analgésicas, antihiperalgésicas y antiinflamatorias, disminuye el consumo de opioides y agentes volátiles, brindando una rápida recuperación de la función intestinal. La lidocaína es un anestésico local tipo amida que actúa bloqueando los canales de sodio dependientes de voltaje (CSDV) en tejidos neuronales, interrumpiendo la transmisión nerviosa. Los efectos indirectos son atribuidos a la modulación de la respuesta inflamatoria ocasionada por el estrés quirúrgico como a la menor exposición a opioides y agentes volátiles ${ }^{(20)}$. Los efectos directos son mediados por acciones moleculares específicas que son ejercidas sobre células cancerígenas. Las células tumorales expresan canales de sodio dependientes de voltaje (CSDV) en una gran variedad de carcinomas, incluyendo el de mama, cuello uterino, colon, pulmón (células pequeñas, células no pequeñas y mesotelioma), piel, ovario y próstata. In vitro, la activación de los canales de sodio dependientes de voltaje ha demostrado aumentar su poder metastásico debido a una mayor motilidad e invasión. De esta manera, las células tumorales se comportan como «eléctricamente excitables», tornándose hiperactivas y adquiriendo agresividad. Este fenómeno se conoce como CELEX, por cell excitability, y representa una hipótesis de progresión metastásica tumoral. El uso de anestésicos locales intraquirúrgicos y postquirúrgicos, al bloquear los canales dependientes de voltaje, podría reducir el poder metastásico de las células tumorales, disminuyendo su motilidad e invasividad. En consecuencia, esta acción podría reducir el escape de células tumorales durante el perioperatorio, aumentando la supervivencia de los pacientes y mejorando su calidad de

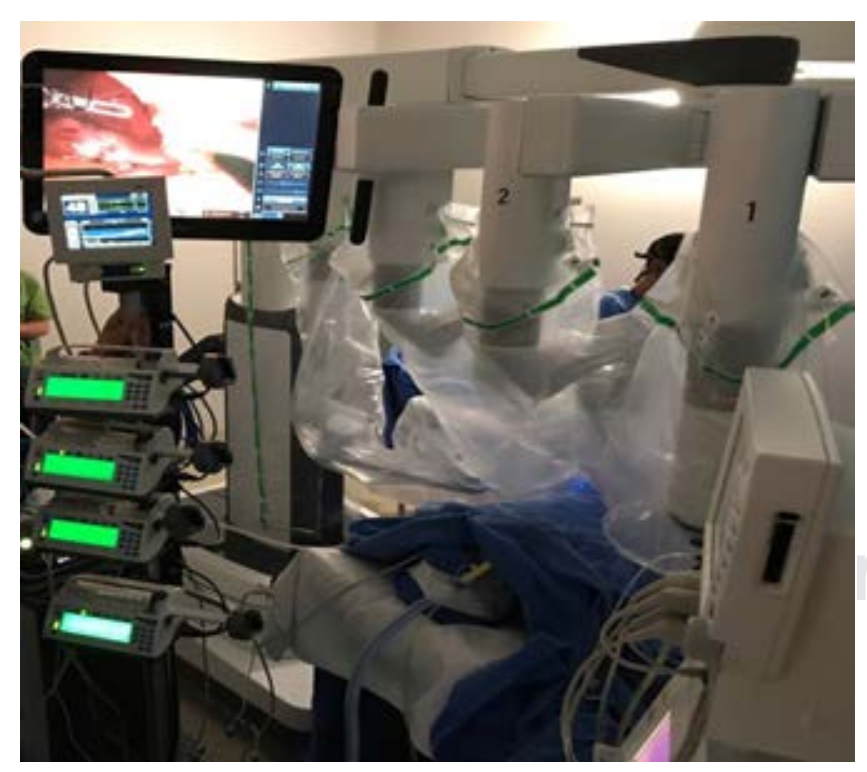

Figura 3: Colocación del robot da Vinci Xi, perfusores manuales para cirugía robótica. vida. Otras acciones diferentes al bloqueo de canales de sodio han sido evidenciadas como posibles propiedades antiproliferativas ${ }^{(21)}$. Estudios in vitro demostraron que la exposición a la lidocaína inhibe la proteína tirosina cinasa Src en células neoplásicas, proteína implicada en la proliferación, migración, invasividad y metástasis tumoral. Estudios farmacocinéticos garantizan adecuadas concentraciones efectivas para los efectos deseados, siendo su toxicidad infrecuente. Entre sus ventajas se destacan su eficacia y efectividad en cirugías abdominales y procedimientos de cirugía ambulatoria, siendo implementada como parte de la terapéutica en protocolos de pronta recuperación postquirúrgica. Estudios actuales analizan su posible acción preventiva sobre el desarrollo de dolor crónico postoperatorio al minimizar la aparición de hiperalgesia inducida por opioides, pudiendo ser utilizada como una técnica analgésica basada en el ahorro de opioides. La lidocaína tiene acciones antiproliferativas sobre células tumorales mediante mecanismos mediados por el bloqueo de canales de sodio dependientes de voltaje (CSDV) y otras dianas moleculares ${ }^{(22)}$.

Comparada con la anestesia inhalatoria, la anestesia intravenosa, y en particular el uso de propofol con edetato, ha demostrado ofrecer beneficios considerables al usarse como agente de inducción y mantenimiento transanestésico. Los agentes inhalados se han asociado a toxicidad hepática y pulmonar y la exposición a pequeñas cantidades de sevofluorano ha demostrado aumentar el riesgo de daño genético equivalente asociado a fumar 11-20 cigarros por día.

El empleo de la anestesia total intravenosa multimodal basada en objetivo al parecer presenta mejores resultados de supervivencia y recurrencia tumorales con la ATIV cuando se emplea la combinación de varios agentes intravenosos para inducción y mantenimiento de la anestesia general, entre ellos, hipnóticos no barbitúricos, opioides, alfa agonistas, anestésicos locales (lidocaína simple), sulfato de magnesio, ketamina y esmolol, entre otros coadyuvantes.

\section{CASO CLÍNICO}

Se realizó cistectomía radical y linfadenectomía pélvica bilateral superextendida laparoscópica asistida por robot Da Vinci Xi bajo ATIV multimodal en modo TCI y manual, con monitoreo objetivo durante más de 15 horas de manejo anestésico (Figura 3).

Masculino de 67 años de edad, ASA 3, con diagnóstico preoperatorio de cáncer de vejiga con escala de estadiaje T3Bn1M1a. Cuenta con los siguientes antecedentes personales relevantes: alérgico a la ranitidina, cirugía en cuello por cornada de toro bajo anestesia general sin complicaciones, cistoscopía bajo anestesia general sin complicaciones. Hipertenso con siete años de evolución en tratamiento actual con irbesartán de 150 mg cada 12 horas vía oral y amlodipino 10 


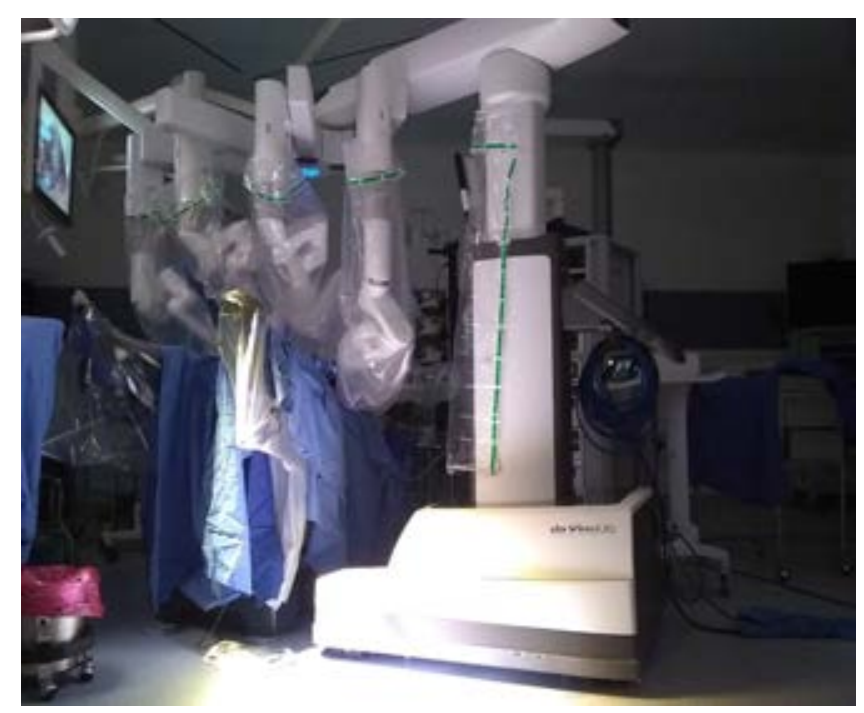

Figura 4. Posición de Trendelenburg forzado 85 grados con robot da Vinci Xi en el Centro Médico Naval.

mg cada 24 horas vía oral; alcoholismo suspendido hace siete años, tabaquismo ocasional con tres cigarros al día suspendido hace dos meses sin presentar otros antecedentes de importancia. Paciente masculino programado de manera electiva para cistectomía radical y linfadenectomía pélvica bilateral superextendida laparoscópica asistida por robot. Actualmente en condiciones adecuadas, estable a nivel cardiorrespiratorio, sin presentar predictores de vía aérea difícil. MALLAMPATI II, BELL HOUSE DORE II, anodoncia parcial con diente centinela lateral superior izquierdo.

Peso del paciente: $64 \mathrm{~kg}$, Altura (mts): 1.65. IMC: 23.52.

Datos de laboratorio: Hb 16.2 mg/dL, Hto 48.6\%, plaquetas 215,000, leucocitos 5.6, glucosa $85.80 \mathrm{mg} / \mathrm{dL}$, urea 12.6 , creatinina 0.82, albúmina $2.8 \mathrm{~g} / \mathrm{dL}$, TP 14.10, INR 1.04, TTP 26.30. Tipo de sangre $\mathrm{O}^{+}$.

Valoración por cardiología: riesgo bajo de eventos cardiovasculares mayores < 1\% (índice de LEE, Destky, Goldman y Guías ESC 2014 de riesgo perioperatorio).

Valoración por geriatría sin contraindicaciones quirúrgicas. RAQ: E3B, RTE: alto, riesgo respiratorio: alto.

Ingresó paciente a sala, despierto, consciente en tiempo, persona y espacio, con presencia de catéter venoso periférico calibre 18 en dorso de mano derecha permeable a solución Ringer lactato con $300 \mathrm{~mL}$; se colocó monitoreo tipo II: $\mathrm{SpO}_{2}$, EKG (DII, V5, AVR), FC, FR, espirometría tipo II, PANI, PAI, NIRS, BIS, TNM, termómetro transnasal, diuresis horaria, sonda orogástrica 18 French.

Signos vitales basales e iniciales: TA 131/87, FC 66X', $\mathrm{SpO}_{2}$ 94\%. BIS: 87-90 Hz, NIRS L73, R77. Se coloca mascarilla facial con $\mathrm{O}_{2}$ a $3 \mathrm{~L} / \mathrm{min}$, sujeto con un soporte facial. Se iniciaron perfusiones continuas intravenosas modo manual de manera simultánea con las bombas Smiths Medical Medex 3500. Lidocaína simple a $1 \mathrm{mg} / \mathrm{kg} / \mathrm{h}$, dexmedetomidina $0.5 \mu \mathrm{g} /$ $\mathrm{kg} / \mathrm{h}$. Iniciando perfusiones de lidocaína simple y dexmedetomidina 20 minutos previo a inducción. Inducción: fentanilo $2 \mu \mathrm{g} / \mathrm{kg}$ iv, en perfusor Mindray Benefusion SP5 Propofol TCI sitio efecto Modelo Schnider $2.4 \mu \mathrm{g} / \mathrm{mL}$ iv, rocuronio $0.6 \mathrm{mg} / \mathrm{kg}$ iv en bolo inicial. Previa desnitrogenización con mascarilla facial a $3 \mathrm{~L} / \mathrm{min}$ por dos minutos. Laringoscopía directa con hoja Macintosh No. 4, intubación de primera intención con tubo endotraqueal armado No. 8.5 con $4 \mathrm{~cm}^{3}$ de neumotaponamiento punta atraumática, se verifica adecuada estancia de tubo endotraqueal mediante auscultación de campos pulmonares a nivel apical y basal, clínica y capnografía. Se fija tubo endotraqueal de manera muy minuciosa y precisa; valoración de Cormack-Lehane II. Se verifica respuesta a nivel pupilar (mióticas, simétricas, concéntricas). Se inserta termómetro transnasal y se coloca sonda orogástrica de 18 French de primera intención corroborando estancia de sonda en cavidad gástrica. Se colocan protecciones en ambos ojos y en la zona de la boca. Parámetros ventilatorios: modo control volumen, VT: 400-450 mL, de manera inicial, FR: 15-20X', Relación I:E: 1:2, PEEP 4-6 $\mathrm{cmH}_{2} \mathrm{O}$, Pmáx: $16-21 \mathrm{~cm}_{2} \mathrm{O}$, $\mathrm{FiO}_{2}$ al $60 \%$. Con el paciente en posición supina y neutra se toma línea arterial radial izquierda con jelco calibre No. 20 permeable a solución de $\mathrm{NaCl}$ de $250 \mathrm{~mL}$ con 1,000 UI de heparina, posteriormente se canaliza vena periférica en antebrazo izquierdo con catéter venoso periférico No. 18, permeable a solución Ringer lactato.

Se colocan cinturones de protección y sujeción en el paciente a nivel torácico con los brazos a los costados del paciente, corroborando de manera exacta los puntos de presión en tórax para no comprometer la mecánica ventilatoria durante el evento quirúrgico. Se posicionan ambas extremidades inferiores en litotomía media, verificando puntos de presión en extremidades superiores, inferiores y tórax en el paciente para después posicionar la mesa quirúrgica en Trendelenburg forzada a $86^{\circ}-87^{\circ}$ de inclinación (Figura 4), realizando el movimiento de la mesa de manera muy lenta verificando estabilidad hemodinámica del paciente, presentando cifras tensionales TA 100/67, FC 61X', $\mathrm{SPO}_{2}$ 99\%, NIRS S62, S59, L77, R79. Ya posicionada la mesa se modifican parámetros ventilatorios con modo de ventilación control volumen con VT 230-250 mL, frecuencia respiratoria de 17-25x', relación I:E 1:2, PEEP 6-8 $\mathrm{cmH}_{2} \mathrm{O}$, Pmáx $20 \mathrm{cmH}_{2} \mathrm{O}, \mathrm{FiO}_{2}$ a 60\% (Figura 5).

Mantenimiento: $\mathrm{FiO}_{2} 60 \%$, propofol TCI a $2.4 \mu \mathrm{g} / \mathrm{mL}$ en sitio efecto equivalente a tasa de perfusión de $2.8 \mu \mathrm{g} / \mathrm{kg} / \mathrm{h}$, fentanilo $3-2 \mu \mathrm{g} / \mathrm{kg} / \mathrm{h}$, dexmedetomidina $0.5 \mu \mathrm{g} / \mathrm{kg} / \mathrm{h}$, lidocaína simple $2 \mu \mathrm{g} / \mathrm{mL}$, rocuronio $0.6 \mu \mathrm{g} / \mathrm{mL}$, sulfato de magnesio en perfusión $7 \mathrm{mg} / \mathrm{kg} / \mathrm{h}$.

Fármacos complementarios: hidrocortisona $100 \mathrm{mg}$ iv, metoclopramida $10 \mathrm{mg}$ iv, clonixinato de lisina $200 \mathrm{mg}$ iv, 
gluconato de calcio 6 g iv, albúmina un frasco $12.5 \%$, paracetamol $1 \mathrm{~g}$ iv, ondansetrón $8 \mathrm{mg}$ iv, tramadol 100 mg iv, cefotaxima $1 \mathrm{~g}$ iv, omeprazol $40 \mathrm{mg}$ iv.

Se mantuvo con cifras tensionales promedio TA 110/60, FC 67-59X', SPO 2 98-99\%, NIRS L73, R70, L 66, R 73, L64, R67. BIS 45-51. TS 0. Temperatura esofágica: $35.8^{\circ} \mathrm{C}$, TNM (TOF 0) cada 15 minutos hasta recuperación espontánea (Figura 6).

Al finalizar el procedimiento quirúrgico se retiran brazos del robot y se corrige posición del paciente de manera lenta hasta posición supina neutra, corroborando que no se presenten cambios hemodinámicos marcados. Previo a la extubación se verifica ausencia de hiperemia en cabeza y cuello, gasométricamente ausencia de acidosis respiratoria, ausencia de edema lingual, ausencia de edema de las conjuntivas, normocapnia en gasometría sanguínea durante la ventilación espontánea, un TNM 90\%. Se aspiran secreciones por sonda orogástrica y se retira la sonda. Se extuba al paciente cumpliendo parámetros ventilatorios adecuados. Se retira línea arterial y se coloca vendaje de $5 \mathrm{~cm}$ en área radial izquierda.

Ingresos: 4,500 mL. Ringer lactato.

Diuresis total 1,120 mL.

Tasa horaria: $1.24 \mathrm{~mL}$.

Tiempo quirúrgico: 920 minutos.

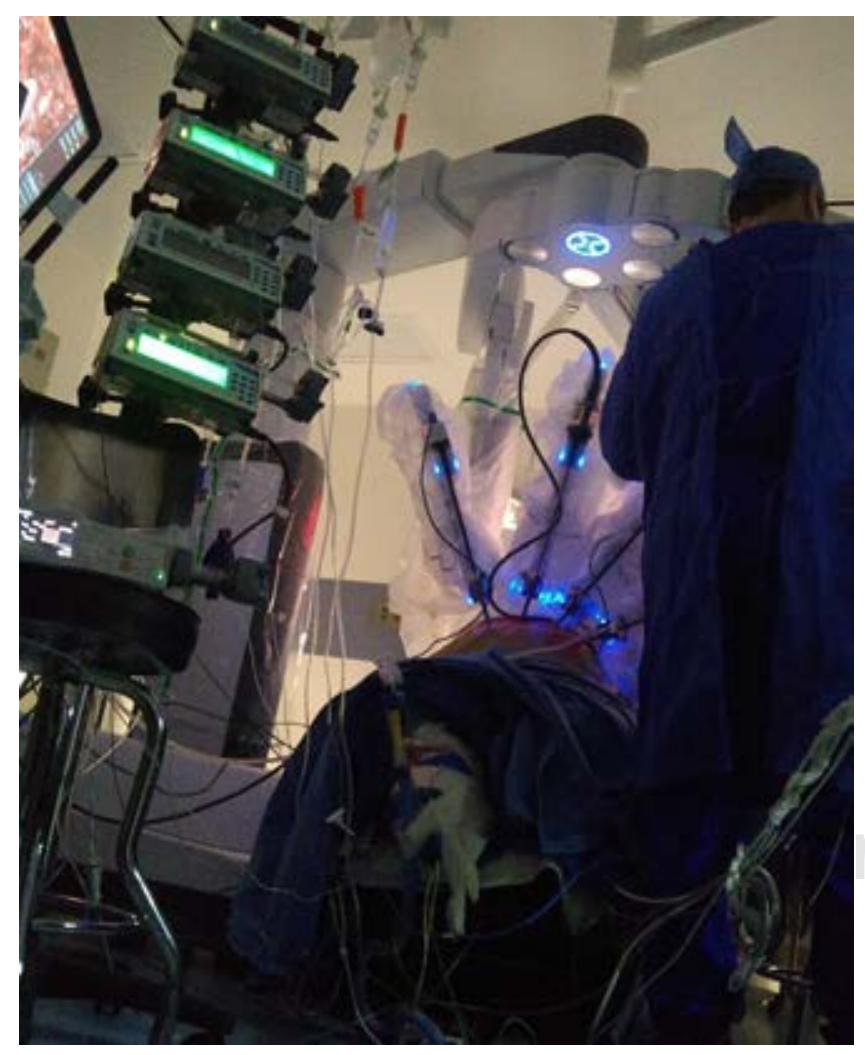

Figura 5: Posición de Trendelenburg forzada con perfusores manuales y perfusor target controlled infusion propofol Schnider a sitio efecto.

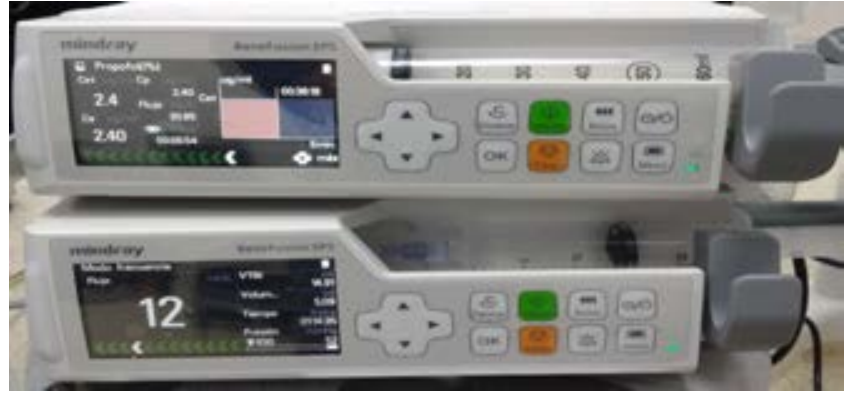

Figura 6: Perfusión target controlled infusion Schnider $2.4 \mu \mathrm{g} / \mathrm{mL}$ a sitio efecto y perfusión manual de sulfato de magnesio $7 \mathrm{mg} / \mathrm{kg} / \mathrm{h}$ promedio.

Tiempo anestésico: 1,020 minutos.

Sangrado: $250 \mathrm{~mL}$.

Gasometría 1:

$\mathrm{Ph} 7.37, \mathrm{PCO}_{2} 28.4, \mathrm{HCO}_{3}$ 16.0, BE -1, Hct 32, Hb 16, Na 135, K 3.12, Ca 0.97, Gluc. 91, Lactato 0.25, temperatura 35.8.

Gasometría 2:

$\mathrm{Ph} 7.35, \mathrm{PCO}_{2} 36.7, \mathrm{HCO}_{3} 22.8$, BE -2.1, Hct 31, $\mathrm{Hb}$ 15.9, Na 136, K 3.61, Ca 1.15, Gluc. 115, lactato 0.90, temperatura 35.4 .

Gasometría 3:

$\mathrm{Ph} 7.35, \mathrm{PCO}_{2} 36.2, \mathrm{HCO}_{3}$ 28.2, BE -2.5, Hct 29, $\mathrm{Hb}$ 15.8, Na 135.2, K 3.63, Ca 1.12, Gluc. 122, lactato 1.18, temperatura 35.

\begin{tabular}{|ccc}
\hline \multicolumn{3}{c}{ Tasa de perfusión propofol-fentanilo. } \\
\hline & Propofol & Fentanilo \\
\hline & $2,400 \mathrm{mg}$ & $700 \mu \mathrm{g}$ \\
\hline Dosis total & $37.5 \mathrm{mg} / \mathrm{kg}$ & $10.93 \mu \mathrm{g} / \mathrm{kg}$ \\
Peso $64 \mathrm{~kg}$ & $0.04 \mathrm{mg} / \mathrm{kg} / \mathrm{min}$ & $0.012 \mu \mathrm{g} / \mathrm{kg} / \mathrm{min}$ \\
Tiempo quirúrgico 920 & $40 \mu \mathrm{gg} / \mathrm{min}$ & $\mathrm{Cp:} 0.0009 \mu \mathrm{g} / \mathrm{mL}$ \\
minutos & $\mathrm{Cp}: 0.0013 \mathrm{mg} / \mathrm{mL}$ & \\
Clearance plasmático & $\mathrm{Cp}: 1.3 \mu \mathrm{g} / \mathrm{mL}$ & \\
Propofol $30 \mathrm{~mL} / \mathrm{kg} / \mathrm{min}$ & & \\
Fentanilo $13 \mathrm{~mL} / \mathrm{kg} / \mathrm{min}$ & &
\end{tabular}

\section{Dosis totales:}

Fentanilo: $700 \mu \mathrm{g}$ iv (tiempo de cierre de bomba una hora antes del término de cirugía)

Propofol con edetato: 12 ampolletas 2,400 mg

Rocuronio: $70 \mathrm{mg}$ iv.

Dexmedetomidina: $200 \mu \mathrm{g}$ iv.

Sulfato de magnesio: $4 \mathrm{~g}$ iv.

Concentraciones plasmáticas finales para un tiempo anestésico estimado de 15.20 horas: fentanilo: $0.0009 \mu \mathrm{g} / \mathrm{mL}$, propofol con edetato $1.3 \mu \mathrm{g} / \mathrm{mL}$, lidocaína simple $1 \mu \mathrm{g} / \mathrm{mL}$, dexmedetomidina $200 \mu \mathrm{g}$ iv.

Se egresa a sala de recuperación, paciente despierto, consciente en sus esferas neurológicas, hemodinámicamente con 
cifras tensionales TA 115/79, FC 68X', $\mathrm{SPO}_{2} 99 \%$. Se coloca monitoreo tipo I, mascarilla facial con oxígeno suplementario a $3 \mathrm{~L} / \mathrm{min}$ al $98 \%$ y cobertor térmico.

Escala de Glasgow 15, Ramsay 2, Aldrete 10, clasificación de Michigan: clase 0. Escala de Richmond 0. Escala de dolor postoperatorio (EVA 0). Escala verbal simple 0.

Permanece el paciente en el área de cuidados postanestésicos una hora aproximadamente acorde a la norma oficial mexicana para la práctica de la anestesiología y es enviado al área de hospitalización.

Al siguiente día durante el pase de visita se encuentra paciente despierto, consciente en sus esferas neurológicas, deambulando, con un EVA de 0, tolerando vía oral, adoptando posición libremente escogida. Se egresa a las 72 horas de postoperatorio del área de hospitalización, sin presentarse eventos adversos, dolor postoperatorio, náusea, vómito, equimosis ni ingurgitación yugular durante su estancia; siendo egresado a su domicilio.

\section{CONCLUSIÓN}

El empleo de anestesia general multimodal por vía intravenosa en este caso permitió un adecuado estado anestésico objetivo basado en que los componentes hipnótico, nociceptivo y hemodinámico posibilitaran que el procedimiento quirúrgico se llevara a cabo mediante una inducción, mantenimiento y recuperación de la conciencia y se controlara con base en una concentración objetivo de manera «fina» mediante el sistema TCI, ajustando la tasa de perfusión y por ende, la titulación con monitoreo cerebral para la conciencia y la oxigenación cerebral, alcanzando y manteniendo una dosificación racional del agente hipnótico. Se demostró que se puede titular el propofol con base en los rangos de concentración reportados en la literatura para el mantenimiento y emersión hasta la recuperación del estado de conciencia, sin evidencia de awareness ni delirio postoperatorio.

La perfusión de fentanilo se llevó a cabo de manera manual mediante la titulación, ajustando la tasa de perfusión de mayor a menor concentración plasmática para atenuar la respuesta a la noxa quirúrgica, y permitir durante el mantenimiento

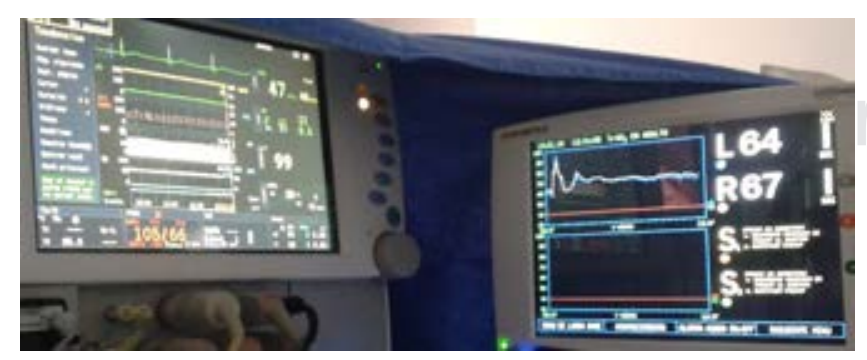

Figura 7: Tendencia hemodinámica durante el transanestésico. anestésico adecuar la concentración basada en la sinergia que ofrecen coadyuvantes del tipo dexmedetomidina, lidocaína y sulfato de magnesio.

Es por esto que el manejo guiado por objetivos específicos basado en la concentración plasmática y sitio efecto que se requiere para alcanzar, mantener y emerger del estado anestésico mediante diversos agentes anestésicos y coadyuvantes es uno de los retos que el médico anestesiólogo debe manejar para ofrecer procedimientos seguros y con calidad en los diversos escenarios clínicos, ofreciendo al paciente anestesia intravenosa con mínima dosis de opioides.

Los conocimientos de farmacología aunados a una adecuada titulación de los agentes anestésicos basada en sus perfiles farmacocinéticos, así como el manejo de herramientas que permitan titular de manera específica y fina a diferentes ritmos de perfusión con base en el estímulo quirúrgico y contar con el monitoreo específico para evaluar momento a momento la respuesta farmacodinamia, permiten adecuar la sinergia entre los diferentes componentes del estado anestésico.

La estabilidad transoperatoria, la disminución en el consumo de fármacos, la analgesia y el confort del paciente son la mejor demostración de los beneficios del empleo multimodal de los agentes anestésicos y sus coadyuvantes, englobando la combinación de dos o más fármacos apoyados en la farmacocinética y la farmacodinamia de los agentes empleados mediante sus interacciones (sinergia y aditividad) (Figura 7).

Las interacciones farmacocinéticas y farmacodinámicas que se producen al hacer combinaciones diversas generan una suma de acciones para producir una respuesta clínica de intensidad variable que dependerá de la vía de administración, la dosis y el número de fármacos empleados, sin olvidar la variabilidad interindividual del paciente. El uso de múltiples agentes antinociceptivos, además de un opioide a bajas concentraciones, crea el efecto ahorrador de estos agentes, demostrando que la perfusión intravenosa de fentanilo en este tipo de técnicas puede ser una herramienta segura, práctica, barata y con mínimos efectos adversos.

En teoría cada agente se dirige a un componente diferente del sistema nociceptivo para que juntos supriman de manera más completa la transmisión nociceptiva, independientemente del tiempo quirúrgico que en este caso fue prolongado: 15 horas con 20 minutos.

Los agentes hipnóticos y coadyuvantes reducen la capacidad de percibir el dolor, y de este modo contribuyen de forma implícita al control de la conciencia y a la antinocicepción, siempre y cuando se ajusten diversas interacciones que permitan alcanzar el efecto sinérgico para adecuar el estado anestésico, siendo obligatorio el empleo de un monitoreo específico para estos componentes con el único fin de ofrecer anestesia adecuada, segura y de calidad ante cualquier escenario clínico. 


\section{REFERENCIAS}

1. Global Cancer Observatory (GCO), Globocan, populations Facts Shets; México.

2. Modi PK, Hollenbeck BK, Oerline M, Weizer AZ, Montgomery JS, Kaffenberger SD, et al. Real-world impact of minimally invasive versus open radical cystectomy on perioperative outcomes and spending. Urology. 2019;125:86-91.

3. Lestar M, Gunnarsson L, Lagerstrand L, Wiklund P, Odeberg-Wernerman S. Hemodynamic perturbations during robot-assisted laparoscopic radical prostatectomy in 45 degrees Trendelenburg position. Anesth Analg. 2011;113:1069-1075.

4. Saito J, Noguchi S, Matsumoto A, Jinushi K, Kasai T, Kudo T. Impact of robot-assisted laparoscopic prostatectomy on the management of general anesthesia: efficacy of blood withdrawal during a steep Trendelenburg position. J Anesth. 2015;29:487-491.

5. Shetty RM, Bellini A, Wijayatilake DS, Hamilton MA, Jain R, Karanth S, Namachivayam A. BIS monitoring versus clinical assessment for sedation in mechanically ventilated adults in the intensive care unit and its impact on clinical outcomes and resource utilization. Cochrane Database of Systematic Reviews. 2018;2:CD011240. doi: 10.1002/14651858.CD011240.pub.

6. Yoshitani K, Kawaguchi M, Ishida K, Maekawa K, Miyawaki H, Tanaka $\mathrm{S}$, et al. Guidelines for the use of cerebral oximetry by near-infrared spectroscopy in cardiovascular anesthesia: a report by the cerebrospinal Division of the Academic Committee of the Japanese Society of cardiovascular Anesthesiologists (JSCVA). J Anesth. 2019;33:167-196.

7. Martin BJ, Buth KJ, Arora RC, Baskett RJ. Delirium: a cause for concern beyond the immediate postoperative period. Ann Thorac Surg. 2012;93:1114-1120.

8. Veiga RJ, García CJ, Orozco MM, Parreño CB, García RJ, Aguayo AJ. Monitoring intraoperative neuromuscular blockade and blood pressure with one device (TOF-Cuff): A comparative study with mechanomyography and invasive blood pressure. Rev Esp Anestesiol Reanim. 2017;64:560-567. doi: 10.1016/j.redar.2017.03.013.

9. Schoen J, Husemann L, Tiemayer C, Lueloh A, Sedemend-ADIB B, Berger KU, Hueppe M, et al. Cognitive function after sevoflurane-vs propofol -based anaesthesia for on-pump cardiac surgery: a randomized controlled trial. Br J Anaesth. 2011;106:840-850.

10. Li R, Liu H, James P. Dilger, Jun Lin. Effect of propofol on breast cancer cell, the. immune system, and patient outcome. BMC Anesthesiol. 2018;18:18-77.
11. Ciechanowicz SJ, Ma D. Anaesthesia for oncological surgery - can it really influence cancer recurrence? Anaesthesia. 2016;71:127-131. Available in: https://doi.org/10.1111/anae.13342.

12. Zheng X, Wang Y, Dong L, Zhao S, Wang L, Chen H, et al. Effects of propofol-based total intravenous anesthesia on gastric cancer: a retrospective study. Oncotargets and therapy. Onco Targets Ther. 2018;11:1141-1148. Available in: http://dx.doi.org/10.2147/ott. s156792.

13. Bonilla CL, Pérez HMA, Torrent A. Efectos de la anestesia en la cirugía oncológica. Rev Anestesiar. 2017;31:21-26.

14. Liang H, Yang CX, Zhang B, Wang HB, Liu HZ, Lai XH. Sevoflurane suppresses hypoxia-induced growth and metastasis of lung cancer cells via inhibiting hypoxia-inducible factor-1 $\alpha$. J Anesth. 2015;29:821-830.

15. Heaney A, Buggy DJ, Can anaesthetic and analgesic techniques affect cancer recurrence or metástasis. Br J Anaesth. 2012;109:i17-i28.

16. Yardeni IZ, Beilin B, Mayburd E, Alcalay Y, Bessler H. Relationship between fentanyl dosage and inmune function in the postoperative period. J Opioid Manag. 2008;4:27-33.

17. Kim S Y. Effects of postoperative pain management on Immune function after laparoscopic resection of colorectal cancer. Medicine (Baltimore). 2016;95:e3602.

18. Wang K, Li C. Efectos de la dexmedetomidina sobre factores inflamatorios, subconjuntos de linfocitos T y expresión de NF- $\mathrm{kB}$ en células mononucleares de sangre periférica en pacientes sometidos a cirugía radical de carcinoma de colon. Oncol Lett. 2018;15:7153-7155.

19. Alday ME, Uña OR, Redondo CF, Criado JA. Magnesio en anestesia y reanimación. Rev Esp Anestesiol Reanim. 2005;52:222-234.

20. Votta-Velis E, Piegeler T, Minshall R, Aguirre J, Beck-Schimmer B, Schwartz D, et al. Regional anaesthesia and cancer metastases: the implication of local anaesthetics. Acta Anaesthesiol Scand. 2013;57:1211-1229. Available in: http://dx.doi.org/ 10.1111/aas.12210.

21. Fraser S, Foo I, Djamgoz M. Local anaesthetic use in cancer surgery and disease recurrence: role of voltage-gated sodium channels? Br J Anaesth. 2014;113:899-902. Available in: http://dx.doi.org/ 10.1093/ bja/aeu221.

22. Piegeler T, Votta-Velis EG, Liu G, Place AT, Schwartz DE, BeckSchimmer B, et al. Antimetastatic potential of amide-linked local anesthetics: Inhibition of lung adenocarcinoma cell migration and inflammatory Src signaling independent of sodium channel blockade. Anesthesiology. 2012;117:548-559. 